\title{
3
}

\section{Gaia and LSST: Their Importance in Binary Star Research}

\author{
LAURENT EYER, NAMI MOWLAVI, ISABELLE LECOEUR-TAIBI, \\ LORENZO RIMOLDINI, BERRY HOLL, MARC AUDARD, \\ SIMON HODGKIN, DAFYDD W. EVANS, LUKASZ WYRZYKOWSI, \\ GEORGE SEABROKE, ANDREJ PRŠA, AND DIMITRI POURBAIX
}

\subsection{Introduction: The Data Revolution in Astronomy}

Observational astronomy is benefitting from modern technological developments and is undergoing profound and deep changes. On one side, the precision/accuracy of the measurements (e.g., the astrometry accuracy, the photometric precision) is improved by several orders of magnitudes, and on the other side the number of sources and number of measurements per source is exploding. We are currently in an era of considerable data growth. Large surveys, such as Gaia and LSST, produce homogeneous data, i.e., the data are acquired with a single set of instruments, and the data need to be processed with software in automated ways. As a consequence, the data processing has become an enormous task, whose cost is of the same order of magnitude as the cost of the contribution of instruments/hardware for ground-based facilities, e.g., for LSST; see Ivezić et al. (2014). In space, the cost of processing is becoming a significant fraction of the hardware, launch and spacecraft operation budget. The Gaia webpage of $\mathrm{ESA}^{1}$ states: 'The total cost of the mission, from the beginning of preliminary studies to the end of operations, is 740 million Euro. This does not include expenditures related to the DPAC ${ }^{2}$ consortium, which are covered by the member states participating in the consortium, not ESA. These costs are currently estimated at around 250 million Euro.'

Besides Gaia and LSST, there are many other future surveys that will have a significant impact on Time Domain Astronomy: HATPI $^{3}$, the new generation of HAT (Bakos et al., 2004), TESS (Ricker et al., 2015), ZTF (Bellm, 2014), and Plato (Rauer et al., 2014), to name a few.

Nonsingle stars, binaries are extremely interesting objects and have an obvious importance in many domains of astronomy. Indeed, they are very common objects in the Universe, they allow to determine directly physical properties of stars such as mass and radius, they can be used as standard candles and they play important roles in star formation and dynamical processes in clusters. However, they add a layer of complexity to the data

\footnotetext{
1 http://www.cosmos.esa.int/web/gaia.

2 Data Processing and Analysis Consortium.

3 https://hatpi.org/.
} 
processing and require significant efforts to process and analyse. Such effort is undertaken in major surveys in order to achieve the detections of binaries, their characterisation, classification and the determination of their astrophysical parameters. From these data, one can then try to infer the properties of the underlying population of nonsingle stars, such as the multiplicity statistics, or the distribution of orbital elements; see, e.g., Duquennoy and Mayor (1991) and Raghavan et al. (2010).

\subsection{Gaia and LSST}

Eyer et al. (2012) gave some descriptions on Gaia and LSST and their impact on binaries. The article contained some comparisons at technical levels and also of precisions/accuracies. The corresponding diagrams were updated in Ivezić et al. (2014). It is often remarked that these two large-scale surveys complement each other very well.

\subsubsection{The Gaia Mission}

The Gaia mission is a cornerstone mission of the Horizon2000+ ESA program. It was selected in 2000, confirmed in 2002 (for a launch no later than 2012) and actually launched in December 2013. The spacecraft performs observations of all the objects brighter than a certain magnitude ${ }^{4}$ limit $\mathrm{G}=20.7$, i.e., about 1.5 billion objects (mostly stars, but also quasars and solar system bodies). The observations consist of astrometric, photometric, spectrophotometric and spectroscopic (for the determination of radial velocities) data. The nominal mission lifetime is five years (each direction of the sky being scanned 70 times on average), from 2014 to 2019, with a possible extension of five additional years. The catalogue of the five-year nominal mission is planned for 2022. If the mission is extended by five years, the final Gaia catalogue is planned for 2027.

The Gaia scanning law: One Field-of-View transit measurement is composed of one measurement of the sky-mapper, followed by nine per-CCD measurements separated by 4.85 seconds each (astrometric field), by BP and RP spectrophotometric measurements, and by RVS (Radial Velocity Spectrometer) measurements. The subsequent Field-of-View transit is done $1 \mathrm{~h} 46 \mathrm{~m}$ later in the majority of cases, possibly followed by another one $4 \mathrm{~h} 14 \mathrm{~m}$ later. Thereafter, there are large gaps of typically three weeks. Statistics of the time sampling are given in Eyer et al. (2017).

Gaia data releases: Initially, five Gaia data releases were planned between 2014 and 2022 (Eyer et al., 2015). However, given the monumental work each data release represents, four releases are currently considered. They are described in the following paragraphs.

The first data release in September 2016 was based on the collection of 14 months of data from end of July 2014 to mid-September 2015. It consisted of (a) position and mean G-magnitude for 1.1 billion stars (Lindegren et al., 2016, and van Leeuwen et al., 4 The Gaia G-band magnitude is a broadband, white-light magnitude in the wavelength range $330-1050 \mathrm{~nm}$ defined by the
telescope transmission and CCD quantum efficiency. 
2017, respectively); (b) five-parameter astrometric solution (position, proper motion and parallax) for 2.1 million stars (Lindegren et al., 2016), a solution that makes use of the Hipparcos-Tycho astrometry and is called TGAS (Tycho-Gaia Astronometric Solution); (c) photometry (light curves) of 3,194 Cepheids and RR Lyrae stars in the direction of the South Ecliptic Pole (direction of the Large Magellanic Cloud) (Clementini et al., 2016; Eyer et al., 2017); (d) position and magnitude of 2,195 quasars (Mignard et al., 2016).

The second data release is planned for April 2018 and is based on 22 months of data. The foreseen products are (a) the five-parameter astrometric solution (position, proper motion and parallax) for more than 1.5 billion stars, (b) photometry ( $\mathrm{G}$ band and integrated BP and RP bands), (c) astrophysical parameters for $\mathrm{G}<17 \mathrm{mag}$, (d) median radial velocity for $\mathrm{G}_{\mathrm{RVS}}<12 \mathrm{mag}$, (e) epoch astrometry for $>10,000$ asteroids and (f) a selected sample of variable stars (though no eclipsing binaries).

The third data release is planned for 2020 and would contain updated astrometry and photometry for all sources, source classification and astrophysical parameters, BP, RP and RVS spectra with astrophysical parameters, radial velocities for $\mathrm{G}_{\mathrm{RVS}}<14$ mag, updated and extended solar system object catalogue and updated and extended variable star catalogue. In addition, the release would include nonsingle stars (astrometric binaries and eclipsing binaries).

The final release for the nominal mission is planned in 2022 and would contain the full astrometric, photometric, radial velocity catalogues, all variable stars and nonsingle stars and source classifications (probabilities), plus multiple astrophysical parameters (derived from BP, RP, RVS and astrometry) for stars, unresolved binaries, galaxies and quasars. A list of exoplanet candidates is also planned. All epoch and transit data for all sources, including all BP/RP/RVS spectra, would be made available.

Gaia Alerts: Gaia also provides a continuous alert stream (Hodgkin et al., 2013) under the responsibility of Cambridge University ${ }^{5}$. As of mid-November 2017, more than 3,800 alerts have been issued, at a constant rate of about six alerts/day for the last 18 months. Only about $17 \%$ of Gaia Alerts are robustly classified, via spectroscopic follow-up from groundbased observatories. The remaining $83 \%$ remain unclassified right now, but the Gaia Alerts team is working to automate the classification of all its remaining alerts (for the future, but also retrospectively) through machine learning approaches. Of the approximately 650 robustly classified alerts, the vast majority (about 80\%) are supernovae, and half of the supernovae are of type Ia.

Gaia Alerts are not limited to the discovery of supernovae. There are suspected Cataclysmic Variables (> 400 alerts are predicted to be CVs), and 18 microlensing event candidates, including the exciting binary microlensing event Gaia16aye. The discovery of Gaia16aye triggered a huge, worldwide campaign of photometric follow-up, resulting in $>17,000$ measurements from 33 telescopes lasting for over one year. There are also objects with abrupt declining brightness such as Young Stellar Objects (32 candidates) and $\mathrm{R}$ CrB stars (six candidates). Quasars are also detected in these alerts (46 candidates). No

\footnotetext{
5 http://gsaweb.ast.cam.ac.uk/alerts/home.
} 
doubt in the thousands of unclassified alerts, there remain many more exciting and unusual events yet to be uncovered and investigated. Gaia also alerted us of a very exciting AM CVn star, which is the first such eclipsing binary with a total eclipse (Campbell et al., 2015).

\subsubsection{The Large Synoptic Survey Telescope (LSST)}

LSST $^{6}$ is a project consisting of an 8-meter ground-based telescope, located in Chile. First light is foreseen in 2020 and regular observations will start in 2022. LSST will detect objects as faint as $24.5 \mathrm{mag}$ (single epoch) to $27 \mathrm{mag}$ (with co-added images). The number of observed objects by LSST is staggering, and is estimated to reach 20 billion stars and 20 billion galaxies. The observations will be done in the five Sloan photometric bands, plus a $y$ band. The lifetime of the project is foreseen to be 10 years. During that period, half of the sky will have 825 visits.

The observing strategy of LSST is not definitively fixed and could evolve during the project. The current plan is that there will be two exposures of 15 seconds (or maybe one of 30 seconds). These two exposures are done to detect and remove cosmic rays. Then, three to five days later, the same field is observed, though very probably in a different filter.

\subsubsection{LSST Data Stream}

The impressive numbers given in the previous sections for Gaia seem small when compared to the ones of LSST. These are just stunning. The nightly alert system will consist of a stream of about 10 million time-domain events per night, detected and transmitted to event distribution networks within 60 seconds of observation. The early Data Releases will contain a catalogue of about 37 billion objects (20 billion galaxies, 17 billion stars), about 7 trillion single-epoch detections (sources), produced annually, accessible through online databases. There will also be deep co-added images.

Along with the data are 'Community Services'. They will consist of service and computing resources provided at the Data Access Centres to enable user-specified custom processing and analysis. Software and APIs (application program interfaces) enabling development of analysis codes are also provided.

\subsection{The Detection of Nonsingle Stars}

Nonsingle stars can have signatures in all astronomical fundamental technics (astrometry, photometry, spectroscopy). Therefore, all these techniques are contributing to their possible detection and characterisation. It is fundamental to understand the details of the selection effects so that real properties of samples of objects can be inferred. For example, the observed amplitude for the signal for a circular orbit is independent of the inclination $i$ of

6 For a recent description of LSST, please refer to Ivezić et al. (2017). 


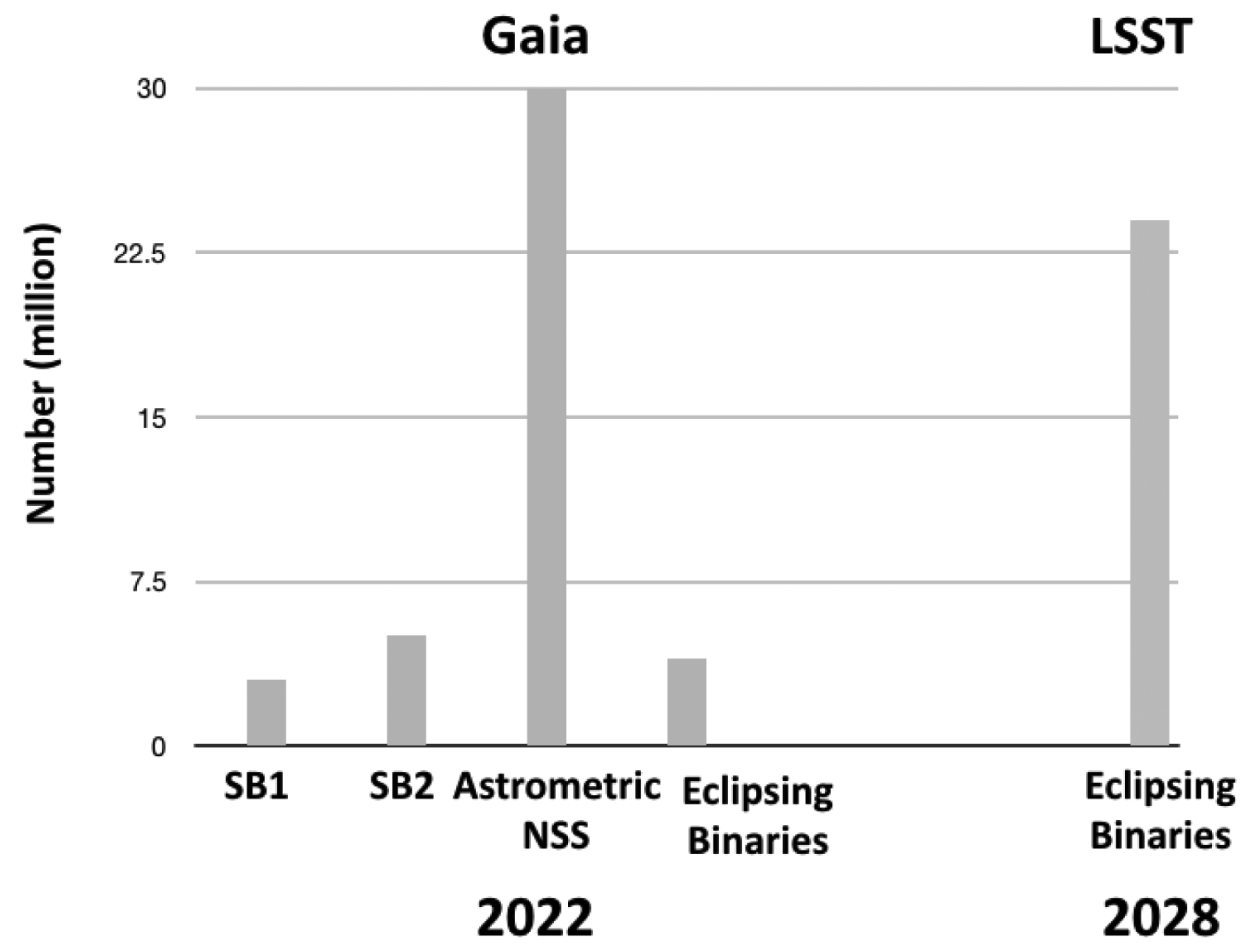

Figure 3.1 Histogram with the estimated number of nonsingle stars to be detected by Gaia and LSST. NSS: nonsingle star, SB1: single-lined spectroscopic binary, SB2: double-lined spectroscopic binary.

the orbit for the astrometry and is a function of $\sin (i)$ for the radial velocity. In the following subsections, we will go through several methods and give estimations (when possible) of the numbers of nonsingle stars detected by Gaia and LSST (see Figure 3.1).

\subsubsection{Astrometric Detection of Binaries}

The position as a function of time of a single star is described by the astrometric solution with five parameters (position, proper motion and parallax), while the astrometric solution of a binary system is fully described by 12 parameters (seven parameters are needed to describe a Keplerian orbit). At best, what can be derived from the orbital parameters are the period, the eccentricity, the inclination, the semimajor axes and the masses (Eyer et al., 2015, table 3). There are intermediate solutions that can be detected, for example a solution with acceleration terms for an orbital period (greatly) exceeding the observational baseline. There are also the Variability-Induced Movers (Wielen, 1996), where an unresolved double star can mimic an apparent astrometric motion if the variability of one component produces an apparent motion of the photocenter. ${ }^{7}$

\footnotetext{
7 Such motion furthermore might be convolved with the true motion of the binary.
} 
An other effect is that the positions of a star measured in distinct photometric bands exhibit some scatter around a central position, consistent with the astrometric precision. When two unresolved stars of very distinct colours are observed with the same distinct photometric bands, the photocentres get scattered around a line joining the two stars. The order of the photocentres further follow the order of the centre of bands. That is what is called a Colour-Induced Displacement double star. That technique was successfully applied with the Sloan Digital Sky Survey (SDSS) (Pourbaix et al., 2004, Pourbaix et al., 2016). Although filter-dependent astrometry was once considered in an early phase of the Gaia mission, it was discarded later on.

Predicted numbers of astrometric binaries from Gaia were presented in Eyer et al. (2013), and reach 30 million stars that are processed as nonsingle stars. There is no estimation of the number of astrometric binaries detected by LSST.

\section{Comoving Pairs}

Another astrometric method to detect binaries is by identifying comoving pairs. It is a way to detect wide binaries. The first Gaia data release was used by several authors to find such binaries, e.g., Andrews et al. (2017), Oh et al. (2017) and Oelkers et al. (2017). From these studies, the number of such detected comoving pairs ranges from 6,000 to 13,000 . The improvement of the astrometric solution from the first Gaia data release to the second will be by several folds. These studies can be repeated, checks on the false detection rates can be estimated and understood and very large numbers of such pairs will be detected.

\subsubsection{Spectroscopic Detection of Binaries}

Spectroscopic binaries can be classified as single-lined (SB1), where several measurements are required to see the doppler shift of the lines, or double-lined (SB2), in which case the two binary components can be directly detected in the spectrum, even in a single measurement. In this latter case, several spectra should be taken to confirm that it is a bound system through the detection of a coherent motion of the two sets of lines.

The RVS on board of Gaia can detect both SB1 and SB2 binaries. It is expected that 150 million stars will have radial velocities. However, to detect binarity, the signal-to-noise level should be high enough. The estimated number of spectroscopic binaries is at the level of about 8 million, with about 5 million being SB2 (Eyer et al., 2013).

It is worth mentioning the Gaia-ESO survey, which consists of 300 nights of observations over five years at the VLT (UVES+ GIRAFFE). This survey is also detecting binaries: Merle et al. (2017) found $354 \mathrm{SB} n$ candidates, where $n$ is the number of components, and more precisely 342 SB2, 11 SB3 and even one SB4.

\subsubsection{Photometric Detection of Binaries}

There are several ways to detect binaries with photometry, such as through their position in the Hertzsprung-Russell diagram or in the colour-colour diagram. Furthermore, the 
photometric light curve can detect eclipses or ellipsoidal variations. There are also indirect ways, such as microlensing events or eclipse/transit timing.

\section{Eclipsing Binaries}

One very classical way to detect binaries is through the detection of the eclipses of the binary system when the orbital plane is favourably aligned with the observer. In recent years, the OGLE survey has provided the largest list to date with more than 450,000 eclipsing and ellipsoidal binary systems towards the Galactic Bulge (Soszyński et al., 2016) and more than 48,000 eclipsing binaries towards the Magellanic System (Pawlak et al., 2016).

From space, the Kepler mission has been a game-changer in the study of eclipsing binaries, with its unprecedented sampling and photometric precision. It allows an extremely detailed description of the light curves. One great surprise of the mission is that $18 \%$ of eclipsing systems observed by Kepler are not strictly regular, showing changes in the eclipses, or even eclipse disappearance, such as in Guinan et al. (2012). The Kepler mission allowed us to estimate the fraction of eclipsing binaries that would be detected by Gaia or LSST. Surprisingly, both surveys would have a detection rate efficiency of about $70 \%$ (Kochoska et al., 2017; Wells et al., 2017). If all the photometric bands of LSST are used, this detection efficiency could (or is expected to) reach $80 \%$.

The estimated number of eclipsing binaries to be detected by Gaia reaches 4 million (Eyer et al., 2013), though this estimate is quite uncertain and varies between 1/2 to 7 million. The expected number of eclipsing binaries to be detected by LSST reaches 24 million (Prša et al., 2011).

\section{Detection of Special Binary Stars}

Here are some additional ways to detect binaries with photometry. It should be noted, that in several of the following cases, the secondary or tertiary star is not necessarily in a stable (bound) orbit. Additional information is therefore required to determine the true nature of boundedness.

Colour-Magnitude diagram: In such a diagram a parallel sequence to the main sequence can be noted (see for example Gaia Collaboration et al., 2016a). It can be observed in clusters or in field stars when the parallax is available. The effect is stronger when the masses of the two stars are similar. When the masses differ, the position of the more massive star is moving towards the upper right in the Colour-Magnitude diagram due to its binarity, and therefore it appears redder.

Colour-Colour diagram: In such a diagram, binaries can be detected because of their location in zones where single stars are not present. Usually it concerns specific pairs; for example, Smolčić et al. (2004) detected with SDSS colours $(u-g, g-r)$ a bridge from white dwarfs to $\mathrm{M}$ stars explained in terms of $\mathrm{M}$ dwarf and white dwarf pairs.

Microlensing: It was realised that binary systems and planetary systems can be detected through microlensing (Mao and Paczynski, 1991). The first system was detected with OGLE data (Udalski et al., 1994). Early warning systems were put in place to first detect 
mircolensing events and then also to detect any deviation from a usual microlensing event so that follow-up observations could be done. A detailed analysis of the light curve can in the best and rare cases give the mass, distance, period of rotation and inclination of the binary lens. More usually, the mass ratio and projected separation in units of Einstein radius can be determined (cf. Jaroszynski et al., 2006). We can note that Gaia has found a spectacular binary event, Gaia16aye ${ }^{8}$ (Wyrzykowski et al., 2017), with multiple caustic crossings. The event is the first binary in the Disk, not in the Bulge. Such events show that one could detect binary black holes like the ones that merged in gravitational wave events.

Orbital variations: Several types of deviations from the strictly periodic light curves of eclipsing binaries can be attributed to a third body. Orbital variations have been studied theoretically by Fabrycky and Tremaine (2007). The variation of the eclipse timings in the Kepler data allowed the detection of 236 systems out of 1,279, where the timing deviation is compatible with the presence of a third body (Conroy et al., 2014).

Ellipsoidal variables: Flux variation as a function of phase does not necessarily imply eclipses; in general, the amount of flux received from a star is proportional to its crosssection. If, due to tidal and rotational distortion of the stellar shape, the cross-section varies, so will the flux. This effect is referred to as ellipsoidal variation, and in close circular orbits it amounts to $\sim 10 \%$ or less. However, the effect can be significantly augmented during periastron flybys of binaries in highly eccentric orbits. These objects feature unique light curves that demonstrate a strong periastron peak and frequent tidally induced pulsations. Because of their resemblance to a cardiogram, this type of objects are called heartbeat stars (Thompson et al., 2012). OGLE found large numbers of ellipsoidal binaries, and Pawlak et al. (2014) identified more than 2,000 such systems towards the Large Magellanic clouds.

Background EBs: When the primary object of interest is not an eclipsing binary, but its light is contaminated by a background eclipsing binary, its light curve will exhibit shallow eclipses. These objects are the main sources of exoplanetary false positives because eclipse depths are compatible with exoplanetary transits and their shapes are often indistinguishable because of the limited signal-to-noise ratio per data point. These are ubiquitous; for example, Abdul-Masih et al. (2016) report a discovery of 285 background EBs in the Kepler dataset.

Other effects: There are quite a few other second-order effects that, thanks to the exquisite precision of modern photometric surveys reaching $\sim \mathrm{ppm}$ accuracy, can be routinely detected. These include Doppler boosting (Bloemen et al., 2012), reflection effect (Derekas et al., 2015), accretion disks (Djurašević et al., 2010) and asteroseismic components (Huber, 2015).

\subsection{The Classification of Binaries}

In this section, we focus on the eclipsing binary classification. One of the great challenges of having large numbers of eclipsing binaries is their automated classification, with the

\footnotetext{
8 See the Gaia Image of the Week of 27 October 2016: http://www.cosmos.esa.int/web/gaia/IoW_20161027.
} 
twofold purposes of (a) studying known classes of binary systems and (b) identifying peculiar binary systems with properties deviating from the ones of known classes. Within the Gaia consortium, the detection and classification of eclipsing binaries are performed by a team based in Geneva (Switzerland) of the Coordination Unit 7 (CU7), while the modelling of the full binary system (orbital and stellar parameters) is performed by a team based in Brussels (Belgium) of the Coordination Unit 4 (CU4) (cf. Siopis and Sadowski, 2012). The full binary system solution is possible only for systems for which enough data, both photometric and spectroscopic, are available.

Four broad categories of binaries are usually considered: detached systems, semidetached systems where one of the stars fills its Roche lobe, contact (or overcontact) systems with potentially a common envelope and ellipsoidal systems. It is, however, rather difficult to provide a classification schema that directly matches these categories based on photometric measurements alone. The 'standard' classification into EA, EB and EW types described in the General Catalogue of Variable Stars, ${ }^{9}$ for example, offers a convenient way for observers to classify eclipsing binary light curves, and explains its still widespread use. But it relies on visual, hence subjective, assessment of the light curve geometry and does not necessarily relate to the afore mentioned categories of binary systems. An attempt in this direction has been proposed by Pojmanski (2002) using Fourier series parameters.

A discrete separation between light curve morphological types is not very realistic, as there is a continuous progression of eclipsing binary properties across the borders from detached to overcontact systems. Matijevič et al. (2012) presented a procedure to classify the light curves with a single parameter that is a measure of the 'detachedness' of the system. The procedure uses locally linear embedding, a general nonlinear dimensionality reduction tool. They successfully apply the method to Kepler eclipsing binaries and show a good correlation of the single parameter with morphology type.

An additional step can be taken with an automated procedure to identify the main components in the light curves of eclipsing binaries. Mowlavi et al. (2017), for example, use Gaussian functions to model the light curve geometry of the eclipses, and sine functions to model the ellipsoidal variability due to tidal effects (the overall model is called the 2-Gaussian model). Applied to the OGLE-III set of eclipsing binaries in the Large Magellanic Cloud, they show how this characterisation of the light curve geometry allows the identification of systems that contain either additional physics (e.g., reflection effects, apsidal motions, discs) or additional variability intrinsic to one or both stars of the binary system. The procedure also provides a set of light curve geometry parameters that are favourable to the statistical analysis of populations of eclipsing binaries. The 2-Gaussian model is currently adopted by CU7 for the processing of Gaia eclipsing binary light curves; see Figure 3.2.

Additional modern classification techniques were proposed in the literature using, for example, a combination of the t-distributed stochastic neighbour embedding (t-SNE)

9 www.sai.msu.su/gcvs/gcvs. 

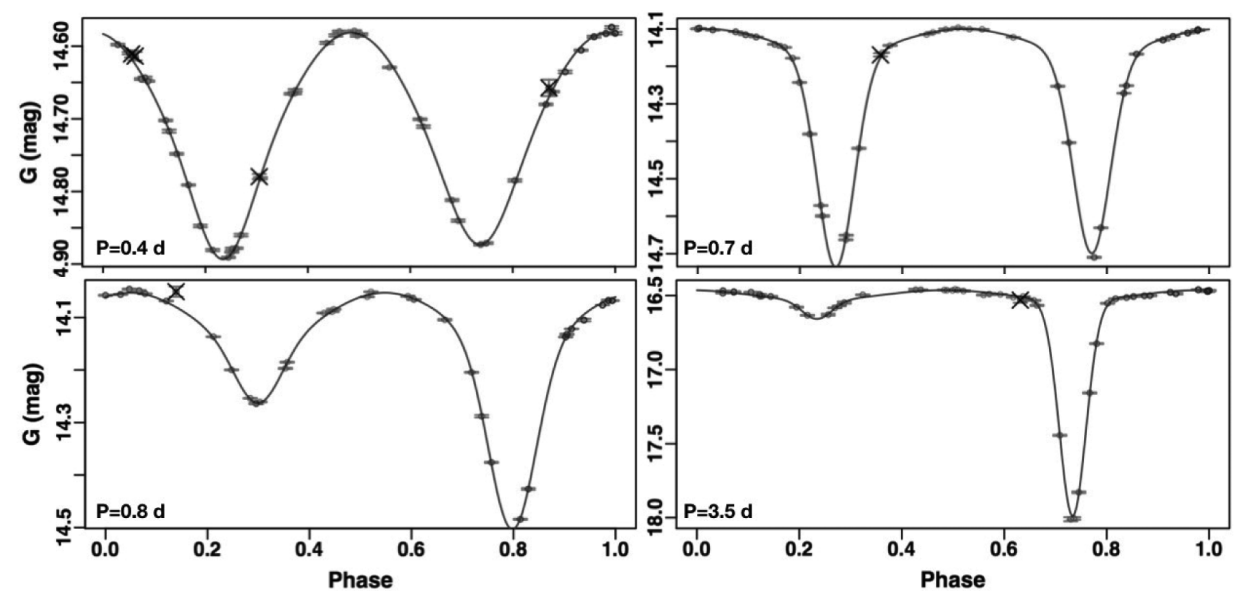

Figure 3.2 Four examples of eclipsing binary light curves of Gaia data fitted with the 2-Gaussian model.

and density-based spatial clustering of applications with noise (DBSCAN) algorithms (Kochoska et al., 2017) or machine learning algorithms (e.g., Süveges et al., 2017).

\subsection{The Characterisation of Binaries}

There are many characteristics that are of interests to binary systems. Examples include the binary fraction among different populations of stars as a function of mass, metallicity intervals, evolution status (dwarf/giant) or their underlying group (halo/disc stars, globular/ open cluster, etc.). It is also interesting to determine the orbital properties, such as period, semimajor axis distribution, mass ratio, etc. Such an exploitation just started with the first data release of Gaia: Boffin and Pourbaix (2018) show the mass-ratio distribution of spectroscopic binaries along the main sequence.

The physical characteristics of binaries can also be compared to those obtained from stellar evolution and pulsation models.

There are fundamental warnings in such studies: there is a need to understand the selection effects of the different star samples, the statistical analysis should be very carefully done especially when using the parallax (there have been numerous misuses of parallaxes in the literature) and there are degeneracies that are sometimes difficult to lift (such as temperature versus extinction).

\subsection{Conclusions}

Binary stars will be one of the topics that is and will be extremely impacted by the Gaia and LSST surveys. The estimated numbers of new detected binaries presented in this chapter are going to reach in total many tens of millions. This data growth is exceptional. In this 
context, it is very relevant to get informed about the data products at each stage of the releases and to be prepared for their scientific exploitation. It is also important to understand some technical details of these surveys to determine their possible limitations, selection functions and statistical properties.

\section{Acknowledgements}

We are indebted to Professor Željko Ivezić (University of Washington), Project Scientist of LSST, who provided material for this contribution.

\section{References}

Abdul-Masih, M., Prša, A., Conroy, K., et al. 2016. Kepler Eclipsing Binary Stars. VIII. Identification of False Positive Eclipsing Binaries and Re-Extraction of New Light Curves. AJ, 151, 101.

Andrews, J. J., Chanamé, J., and Agüeros, M. A. 2017. Wide Binaries in Tycho-Gaia: Search Method and the Distribution of Orbital Separations. MNRAS, 472, 675.

Bakos, G., Noyes, R. W., Kovács, G., et al. 2004. Wide-Field Millimagnitude Photometry with the HAT: A Tool for Extrasolar Planet Detection. PASP, 116, 266.

Bellm, E. 2014. The Zwicky Transient Facility. The Third Hot-Wiring the Transient Universe Workshop. Edited by P. R. Wozniak, 27.

Bloemen, S., Marsh, T. R., Degroote, P., et al. 2012. Mass Ratio from Doppler Beaming and Rmer Delay versus Ellipsoidal Modulation in the Kepler Data of KOI-74. MNRAS, 422, 2600.

Boffin, H. M. J., and Pourbaix, D. 2018. The Mass-Ratio Distribution of Spectroscopic Binaries along the Main-Sequence. Recio-Blanco, A., de Laverny, P., Brown, A. G. A., and Prusti, T. (eds), IAU Symp., 330, 339.

Campbell, H. C., Marsh, T. R., Fraser, M., et al. 2015. Total Eclipse of the Heart: The AM CVn Gaia14aae/ASSASN-14cn. MNRAS, 452, 1060.

Clementini, G., Ripepi, V., Leccia, S., et al. 2016. Gaia Data Release 1: The Cepheid and RR Lyrae Star Pipeline and Its Application to the South Ecliptic Pole Region. A\&A, 595, 133.

Conroy, K. E., Prša, A., Stassun, K. G., et al. 2014. Kepler Eclipsing Binary Stars. IV. Precise Eclipse Times for Close Binaries and Identification of Candidate Three-Body Systems. AJ, 147, 45.

Derekas, A., Németh, P., Southworth, J., et al. 2015. A New sdO+dM Binary with Extreme Eclipses and Reflection Effect. ApJ, 808, 179.

Djurašević, G., Latković, O., Vince, I., and Cséki, A. 2010. Accretion Disc in the Eclipsing Binary AU Mon. MNRAS, 409, 329.

Duquennoy, A., and Mayor, M. 1991. Multiplicity among Solar-Type Stars in the Solar Neighbourhood. II. Distribution of the Orbital Elements in an Unbiased Sample. A\&A, 248, 485.

Eyer, L., Dubath, P., Mowlavi, N., et al. 2012. The Impact of Gaia and LSST on Binaries and Exoplanets. From Interacting Binaries to Exoplanets: Essential Modeling Tools, Proceedings of the International Astronomical Union. Edited by M. T. Richards and I. Hubeny. IAU Symp., 282, 33. 
Eyer, L., Holl, B., Pourbaix, D., et al. 2013. The Gaia Mission. Central European Astrophysical Bulletin, 37, 115.

Eyer, L., Mowlavi, N., Evans, D. W., et al. 2017. Gaia Data Release 1: The Variability Processing \& Analysis and Its Application to the South Ecliptic Pole Region. ArXiv e-prints, 1702.03295.

Eyer, L., Rimoldini, L., Holl, B., et al. 2015. The Gaia Mission, Binary Stars and Exoplanets. Living Together: Planets, Host Stars and Binaries. Edited by S. M. Rucinski, G. Torres and M. Zejda. ASPC, 496, 121.

Fabrycky, D., and Tremaine, S. 2007. Shrinking Binary and Planetary Orbits by Kozai Cycles with Tidal Friction. ApJ, 669, 1298.

Gaia Collaboration, Brown, A. G. A., Vallenari, A., et al. 2016a. Gaia Data Release 1. Summary of the Astrometric, Photometric, and Survey Properties. $A \& A, 595,2$.

Gaia Collaboration, Prusti, T., de Bruijne, J. H. J., et al. 2016b. The Gaia Mission. A\&A, 595, 1.

Gaia Collaboration, van Leeuwen, F., Vallenari, A., et al. 2017. Gaia Data Release 1. Open Cluster Astrometry: Performance, Limitations, and Future Prospects. A\&A, 601, 19.

Guinan, E., Bonaro, M., Engle, S., and Prša, A. 2012. Eclipsing Binaries That Don't Eclipse Anymore: The Strange Case of the Once (and Future?) Eclipsing Binary QX Cassiopeiae. JAAVSO, 40, 417.

Hodgkin, S. T., Wyrzykowski, L., Blagorodnova, N., and Koposov, S. 2013. Transient Astronomy with the Gaia Satellite. Phil. Trans. Royal Soc. of London Series A, 371, 20120239.

Huber, D. 2015. Asteroseismology of Eclipsing Binary Stars. in Giants of Eclipse: The $\zeta$ Aurigae Stars and Other Binary Systems, ASSL, 408, 169.

Ivezić, Ž., Kahn, S. M., and Eliason, P. 2014. The Gaia-LSST Synergy. EAS Publ. Ser., 67, 211.

Ivezić, Ž., Connolly, A. J., and Jurić, M. 2017. Everything We'd Like to Do with LSST Data, but We Don't Know (Yet) How: Astroinformatics. IAU Symp., 325, 93.

Jaroszynski, M., Skowron, J., Udalski, A., et al. 2006. Binary Lenses in OGLE-III EWS Database: Season 2004. Acta Astron., 56, 307.

Kochoska, A., Mowlavi, N., Prša, A., et al. 2017. Gaia Eclipsing Binary and Multiple Systems: A Study of Detectability and Classification of Eclipsing Binaries with Gaia. $A \& A, \mathbf{6 0 2}, 110$.

Lindegren, L., Lammers, U., Bastian, U., et al. 2016. Gaia Data Release 1. Astrometry: One Billionj Positions, Two Million Proper Motions and Parallaxes. A\&A, 595, 4.

Mao, S., and Paczynski, B. 1991. Gravitational Microlensing by Double Stars and Planetary Systems. ApJ, 374, L37.

Matijevič, G., Prša, A., Orosz, J. A., et al. 2012. Kepler Eclipsing Binary Stars. III. Classification of Kepler Eclipsing Binary Light Curves with Locally Linear Embedding. AJ, $143,123$.

Merle, T., Van Eck, S., Jorissen, A., et al. 2017. The Gaia-ESO Survey: Double, Triple and Quadruple-Line Spectroscopic Binary Candidates. A\&A, 608, 34.

Mignard, F., Klioner, S., Lindegren, L., et al. 2016. Gaia Data Release 1. Reference Frame and Optical Properties of ICRF Sources. $A \& A, \mathbf{5 9 5}, 5$.

Mowlavi, N., Lecoeur-Taïbi, I., Holl, B., et al. 2017. Gaia Eclipsing Binary and Multiple Systems. Two-Gaussian Models Applied to OGLE-III Eclipsing Binary Light Curves in the Large Magellanic Cloud. A\&A, 606, 92. 
Oelkers, R. J., Stassun, K. G., and Dhital, S. 2017. Gaia Assorted Mass Binaries Long Excluded from SLoWPoKES (GAMBLES): Identifying Ultra-Wide Binary Pairs with Components of Diverse Mass. AJ, 153, 259.

Oh, S., Price-Whelan, A. M., Hogg, D. W., Morton, T. D., and Spergel, D. N. 2017. Comoving Stars in Gaia DR1: An Abundance of Very Wide Separation Comoving Pairs. AJ, 153, 257.

Pawlak, M., Soszyński, I., Pietrukowicz, P., et al. 2014. Period-Luminosity Relations for Ellipsoidal Binary Stars in the OGLE-III Fields of the Large Magellanic Cloud. Acta Astron., 64, 293.

Pawlak, M., Soszyński, I., Udalski, A., et al. 2016. The OGLE Collection of Variable Stars: Eclipsing Binaries in the Magellanic System. Acta Astron., 66, 421.

Pojmanski, G. 2002. The All Sky Automated Survey. Catalog of Variable Stars. I. 0 h - 6 hQuarter of the Southern Hemisphere. Acta Astron., 52, 397.

Pourbaix, D., Ivezić, Ž., Knapp, G. R., Gunn, J. E., and Lupton, R. H. 2004. Color-Induced Displacement Double Stars in SDSS. $A \& A, \mathbf{4 2 3}, 755$.

Pourbaix, D., Knapp, G. R., Gunn, J. E., et al. 2016. Robust Detection of CID Double Stars in SDSS. A\&A, 591, 96.

Prša, A., Pepper, J., and Stassun, K. G. 2011. Expected Large Synoptic Survey Telescope (LSST) Yield of Eclipsing Binary Stars. AJ, 142, 52.

Raghavan, D., McAlister, H. A., Henry, T. J., et al. 2010. A Survey of Stellar Families: Multiplicity of Solar-Type Stars. ApJ $S, \mathbf{1 9 0}, 1$.

Rauer, H., Catala, C., Aerts, C., et al. 2014. The PLATO 2.0 Mission. Experimental Astronomy, 38, 249.

Ricker, G. R., Winn, J. N., Vanderspek, R., et al. 2015. Transiting Exoplanet Survey Satellite (TESS). Journal of Astronomical Telescopes, Instruments, and Systems, $\mathbf{1}$, 014003.

Siopis, C., and Sadowski, G. 2012. Towards an Automated Processing of Gaia Eclipsing Binaries. In Proceedings of the Workshop 'Orbital Couples: Pas de Deux in the Solar System and the Milky Way'. Edited by F. Arenou, D. Hestroffer, 59.

Smolčić, V., Ivezić, Ž., Knapp, G. R., et al. 2004. In A Second Stellar Color Locus: A Bridge from White Dwarfs to M Stars. ApJ L, 615, L141.

Soszyński, I., Pawlak, M., Pietrukowicz, P., et al. 2016. The OGLE Collection of Variable Stars: Over 450000 Eclipsing and Ellipsoidal Binary Systems Toward the Galactic Bulge. Acta Astron., 66, 405.

Süveges, M., Barblan, F., Lecoeur-Taïbi, I., et al. 2017. Gaia Eclipsing Binary and Multiple Systems: Supervised Classification and Self-Organizing Maps. A\&A, 603, 117.

Thompson, S. E., Everett, M., Mullally, F., et al. 2012. A Class of Eccentric Binaries with Dynamic Tidal Distortions Discovered with Kepler. ApJ, 753, 86.

Udalski, A., Szymanski, M., Mao, S., et al. 1994. The Optical Gravitational Lensing Experiment: OGLE No. 7: Binary Microlens or a New Unusual Variable? ApJ, 436, L103.

van Leeuwen, F., Evans, D. W., De Angeli, F., et al. 2017. Gaia Data Release 1. The Photometric Data. A\&A, 599, 32.

Wells, M., Prša, A., Jones, L., and Yoachim, P. 2017. Initial Estimates on the Performance of the LSST on the Detection of Eclipsing Binaries. PASP, 129, 065003.

Wielen, R. 1996. Searching for VIMs: An Astrometric Method to Detect the Binary Nature of Double Stars with a Variable Component. A\&A, 314, 679.

Wyrzykowski, L., Mroz, P., Rybicki, K., et al. 2017. Gaia16aye Binary Microlensing Event Is Rising for the 5th time. Astronomer's Telegram, 10341. 